Foundations of Physics Letters, Vol. 9, No. 1, 1996, pages 25-42

\title{
DOES THE UNIVERSE IN FACT CONTAIN ALMOST NO INFORMATION?
}

\author{
Max Tegmark \\ Max-Planck-Institut für Physik \\ Föhringer Ring 6 \\ D-80805 München, Germany \\ max@mppmu.mpg.de
}

Received June 2, 1995; revised November 10, 1995

At first sight, an accurate description of the state of the universe appears to require a mind-bogglingly large and perhaps even infinite amount of information, even if we restrict our attention to a small subsystem such as a rabbit. In this paper, it is suggested that most of this information is merely apparent, as seen from our subjective viewpoints, and that the algorithmic information content of the universe as a whole is close to zero. It is argued that if the Schrödinger equation is universally valid, then decoherence together with the standard chaotic behavior of certain non-linear systems will make the universe appear extremely complex to any self-aware subsets that happen to inhabit it now, even if it was in a quite simple state shortly after the big bang. For instance, gravitational instability would amplify the microscopic primordial density fluctuations that are required by the Heisenberg uncertainty principle into quite macroscopic inhomogeneities, forcing the current wavefunction of the universe to contain such Byzantine superpositions as our planet being in many macroscopically different places at once. Since decoherence bars us from experiencing more than one macroscopic reality, we would see seemingly complex constellations of stars etc., even if the initial wavefunction of the universe was perfectly homogeneous and isotropic.

Key words: complexity, chaos, symmetry-breaking, decoherence.

Online at http://www.mpa-garching.mpg.de/ max/nihilo.htm (faster from Europe) and at http://astro.berkeley.edu/ max/nihilo.htm (faster from the US). 


\section{INTRODUCTION}

One of the most striking features of the universe we inhabit is its complexity. When going swimming after setting up an over-night hydrodynamics batch job on the computer, it is easy to start marveling over the system of currents, waves and vortices in the pool, and ask oneself silly questions like "how on earth can nature calculate all this in real time?". Quite apart from the fact that many of the non-linear partial differential equations that nature appears to integrate with ease are numerically ill-posed as initial value problems, exhibiting chaotic behavior, the sheer number of bytes required to store the initial data for a system such as Niagara falls at a reasonable resolution are mind-boggling. Indeed, if we were to take classical General Relativity seriously, then space is a continuum locally isomorphic to $\mathbf{R}^{3}$, which means that to describe the location of something we need to specify three real numbers. Of course, this already constitutes an infinite about of information - to specify a generic real number completely would be equivalent to specifying an infinite number of seemingly random decimals. Likewise, to specify a quantity such as the electric field $\mathbf{E}$ at even a single point would involve specifying real numbers, i.e., an infinite amount of information. This has disturbed many authors in the past, and has been one of the motivations for attempts to replace continuum physics by some discrete alternative, e.g. [1,2, 2, 3].

In this paper, we will instead take the viewpoint of the old phrase "it's just a figment of your imagination", and argue that continuum physics can be maintained without involving infinite quantities of information, and indeed without invoking any new physics whatsoever. The argument will basically go as follows.

- The wave function of the universe shortly after the big bang had some quite simple form, which can be described with very little algorithmic information.

- By the Heisenberg uncertainty principle, this initial state involved micro-superpositions, microscopic quantum fluctuations in the various fields.

- The ensuing time-evolution involved non-linear elements that exhibited chaotic behavior (such as the well-known gravitational instability that is held responsible for the formation of cosmic large-scale structure), which amplified these micro-superpositions into macrosuperpositions.

- In the no collapse version of quantum mechanics, the current wavefunction of the universe is thus a superposition of a large number of states that are macroscopically extremely different (Earth forms here, Earth forms one meter further north, etc).

- Since macroscopic objects inevitably interact with their surroundings, 
the well-known effects of decoherence will prevent self-aware subsets of the universe (such as us) from perceiving such macro-superpositions.

- The net result is that although the wavefunction of the universe contains almost no algorithmic information (we can specify it by simply giving the initial wave function and the Hamiltonian), and will retain for instance translational and rotational symmetry from the initial data, we will experience an asymmetric universe that appears extremely complex.

In other words, the ensemble of all branches in the current macro-superposition is simple to describe, but to describe any one element of the ensemble (because of decoherence, that is all we will observe), requires an enormous amount of information. This is much like the fact that one needs very little information to describe a real-valued random variable with a simple probability distribution, but an infinite amount of information to describe any one realization of it. We will now discuss the elements of this argument in somewhat greater detail.

\section{ALGORITHMIC INFORMATION AND INITIAL CONDITIONS}

The algorithmic information content [4] (also known as the information content, the algorithmic complexity or algorithmic randomness) of a bit string $s$ is defined as the length (in bits) of the shortest program for a universal computer that produces $s$ as output. For instance, the information content of a string of $10^{9}$ random bits would be about $10^{9}$ bits, whereas that of a string of $10^{9}$ zeroes would be quite small. Subtle details such as assumed capabilities of the "universal computer" are discussed in detail in the literature, and are irrelevant to the present discussion. The above definition is straightforward to extend to wider classes of objects than bit strings. Integers are of course bit strings when written in binary form, and the definition of the information content of a real number is analogous. The algorithmic information content of a wavefunction $\psi$ is customarily (e.g., [17]) defined as the length of the shortest computer program that given any point $\mathbf{r}$ in the configuration space and a prescribed accuracy $\epsilon$ will evaluate $\psi(\mathbf{r})$ to this accuracy. Similarly, the information definition is readily widened to encompass the other mathematical objects that will recur in this paper.

As an example, consider an image of the Mandelbrot set. We can equivalently think of it as a scalar field in the complex plane, whose value determines the color of the image at each point (say black or white). To the lay observer, it might appear to contain a vast and perhaps even infinite amount of information. However, since we can describe the Mandelbrot set it in a single sentence, simply as the set of points $c$ in the complex plane for which the iteration $z \mapsto z^{2}+c$ will not diverge when we start with $z=0$, a short 
program can determine whether a given point belongs to the set or not, and the algorithmic information content of the image is thus correspondingly small.

\subsection{The Sense in Which the Whole is Less than a Sum of its Parts}

In the case of the Mandelbrot set, the source of the discrepancy between apparent and actual (algorithmic) information content was chaos, caused by nonlinear dynamics. We will return to this in Sec. 3, as this is one of the two things that we will argue causes the abundance of apparent information around us. The second source is related to quantum symmetry breaking, and will be elaborated upon in Sec. 4. Loosely speaking, the apparent information content rises when we restrict our attention to one particular element in an ensemble, thus losing the symmetry and simplicity that was inherent in the totality of all elements taken together. A uniform probability distribution on the unit interval $[0,1]$ has a very small algorithmic information content, since the distribution function simply equals unity on this interval and vanishes outside of it. Now let us draw one number from this distribution. It can be written out with infinitely many decimals, so to describe it requires an infinite amount of algorithmic information unless we are lucky enough to draw something simple like a rational number, $1 / \sqrt{2}, 1 / \pi$, etc., and this rarely happens - indeed, it is easy to prove that the probability of drawing a number with a finite amount of algorithmic information is zero.

In short, any one realization contains more information than the probability distribution itself. Similarly, any one generic real number between zero and one contains more information than the totality of all of them the algorithmic information content of a single number is infinite, whereas the entire set $[0,1]$ can be generated in lexicographical order by quite a simple computer program [16].

\subsection{The Initial State of the Universe}

In modern cosmology, the field of density fluctuations in the universe (see e.g. [19]) is usually modeled as a random field $\delta(\mathbf{r})$, and it is often assumed that this field was homogeneous, isotropic and Gaussian early on, long before non-linear structures such as galaxies formed. This means that the entire field (or rather the entire ensemble of all fields) can be completely described by merely one single function of one variable, $\xi(r)$, the correlation function, which specifies the correlation of the field values at two points separated by a distance $r$. If this function were given by a simple analytic

\footnotetext{
${ }^{1}$ Any computer program of finite length can be thought of as a rational number between 0 and 1 by interpreting its bits as binary decimals preceded by " 0. .". Since the set of all rational numbers on $[0,1]$ has Lebesgue measure zero, the result follows.
} 
expression, then this particular aspect of the initial data would thus have a very small algorithmic information content. There is of course a catch here: the ensemble of all random fields is merely a convenient statistical concept, and few cosmologists would take this literally enough to believe that there are in fact infinitely many universes with the same correlation function, us merely being ignorant as to which one we happen to inhabit. However, as will be argued in the two following sections, the no-collapse interpretation of quantum mechanics provides a formalism which is in many ways similar to that of random fields, and in addition is supposed to describe actual macroscopic realities that exist in superposition.

A familiar example is the vacuum state of any field theory, which could be interpreted as a superposition of an infinite number of different field configurations. Although this is usually not useful when computing half-lives, cross sections etc., such a vacuum can be characterized in a way quite analogous to that in which random fields are defined, the only major difference being that all probabilities are replaced by complex-valued amplitudes. If the vacuum is homogeneous and isotropic, the description simplifies greatly just as in the above-mentioned random field case. The key point is that the algorithmic information content of the vacuum state of any field theory that we can define is finite, since by merely defining the field theory, we automatically define its vacuum. Although we know virtually nothing about quantum gravity and the state of the universe less than a Planck time after the big bang, it does not appear particularly outrageous to postulate that the universe was once in something resembling a vacuum state of a more complete field theory than we presently know of (incorporating quantum gravity), or at least in some sort of state that would be equally simple to describe. For the rest of our argument, we will simply assume that state of the universe at some early time exhibited so much symmetry that it contained only a small and finite amount of algorithmic information.

\section{CHAOS AND SYMMETRY BREAKING}

Classical physics provides us with an abundance of familiar examples where the apparent information content of a system rises although the algorithmic information content does not, because of chaotic behavior. Water approaching Niagara Falls and a window hit by a football are two familiar examples. In both of these cases, the dynamics amplifies microscopic differences in the initial data into visible macroscopic differences. Here we will center our discussion around an example of gravitational instability.

\subsection{The Classical Cosmological Density Field}

Assuming that the laws of physics that govern the time-evolution are known, the algorithmic information content of the final state is of course not much greater than that of the initial state, since the program that specifies the 
final state can can simply contain the program that computes the initial state together with the appropriate numerical routines (for integration of the partial differential equations of nature, say). The fact that the time required for the computation might be prohibitive is irrelevant here, since algorithmic information refers only to the size of the computer program, not to the time it takes to run it.

As an example, let us assume that we know the field of cosmological density fluctuations at an early time as well as the physics that describes its time evolution. Let us also assume that the initial data are those of a standard cosmological structure formation scenario, i.e., that the initial fluctuations are very small, corresponding to a nearly uniform density, with an appropriate power spectrum (see, e.g., 19 ). To keep things simple, we can assume that all matter is collisionless and that no other forces than classical gravity are at work (including further elements of realism would of course make the system even more chaotic). This uniquely specifies the state of the matter distribution today, and we know from numerical simulations that despite the simplicity of the initial data, this state would be extremely complex, with highly non-linear clumping on a wide range of scales. In other words, gravity alone creates apparent complexity of the type we mentioned when discussing the Mandelbrot set. More precisely, gravitational dynamics causes chaotic behavior, in the sense that a pair of very similar initial data sets could produce results that are macroscopically different - recall that gravitational dynamics exhibits chaotic behavior even in the much simpler case of the three body problem.

\subsection{The Quantum Case}

To be able to appeal to the example of gravitational dynamics for our main argument, we need to take this one step further, beyond classical physics. If the initial state corresponded to exactly uniform density, then so would the final state. Since the real world has a non-zero Planck constant, the actual initial state in our simple gravity problem is of course described by a wavefunctional, $\psi$, that associates a complex amplitude to each configuration of the field of density fluctuations, $\delta(\mathbf{r})$, and the time-evolution is governed not by the classical equations of motion for $\delta(\mathbf{r})$, but by the Schrödinger equation for $\psi$. As is well known, classical statistical mechanics, where a probability distribution in phase space evolves over time according to the Liouville equation, exhibits many similarities with quantum statistical mechanics, where a Wigner function evolves in the same phase space [20,21]. In this phase space picture, there are two familiar differences between the classical and quantum-mechanical descriptions:

1. The quantum time evolution is slightly different, except for Hamiltonians that are of quadratic or lower degree in positions and momenta.

2. Certain classically allowed sets of initial data are forbidden by the Heisenberg uncertainty principle. 
Although of great relevance in many microphysical problems [22], the first of these two differences is irrelevant for our present discussion, as the correction terms are small, and moreover have the effect of making the phase-space time-evolution even more non-linear and chaotic. Thus for our purposes, we can get qualitatively correct results by ignoring this first difference, and simply replacing the quantum treatment by the treatment in terms of classical random fields which has become standard in cosmology, and for which we already know the answer. The second difference is the crucial one here, since as we will see, it implies that the initial $\psi$ cannot simply correspond to the trivial solution of $\delta$ being constant with vanishing time derivatives. For a single particle, the uncertainty principle implies that $\Delta x \Delta p \geq \hbar / 2$, which means that the compulsory $p \neq 0$ components will cause a localized wave packet to gradually "spread out". For the familiar textbook problem of a pencil perfectly balanced on its tip, the uncertainty principle analogously forbids the trivial classical solution where the pencil remains balanced forever. Our gravitational instability example is analogous, except that we are dealing with a quantum field $\delta(\mathbf{r})$ with an infinite number of degrees of freedom. The uncertainly principle simply bounds from below the product of $\Delta \delta(\mathbf{r})$ with the spread in the corresponding conjugate momentum, thus forbidding the trivial classical solution with no fluctuations (where $\delta$ retains a constant value throughout space with no spread, corresponding to the balanced-pencil solution).

In summary, the initial field $\delta(\mathbf{r})$ must be in a superposition of having several different values at each point, and will therefore change due to gravity. We thus draw the following conclusion: since any one initial field configuration with small fluctuations will eventually evolve into a messy and lumpy mass distribution, the initial state $\psi$, being a superposition of such states, will evolve into a superposition of messy and lumpy states.

\subsection{Symmetry is Still not Broken}

It should be stressed that although a type of symmetry breaking has occurred for each individual element of the superposition (approximate translational invariance has been broken), the wavefunction $\psi$ retains all the symmetry that it originally had, since gravity itself is translationally and rotationally invariant. If the the initial $\psi$ had exact translational invariance (which means that the one-dimensional probability distribution for $\delta(\mathbf{r})$, the two-dimensional probability distribution for $\left[\delta\left(\mathbf{r}_{1}\right), \delta\left(\mathbf{r}_{2}\right)\right]$, etc. were all invariant under a shifting of the coordinate system), then the resulting wavefunction today will have this same property. Where is all the irregular gravitational clumping hidden? The answer is to be found in all sorts of intricate long-range correlations that did not exist in the initial $\psi$. For an extreme example, suppose that the time-evolution regrouped all the mass into homogeneous lumps the size of Earth. Then the correlation between $\delta\left(\mathbf{r}_{1}\right)$ and $\delta\left(\mathbf{r}_{2}\right)$ would be almost perfect if $\mathbf{r}_{1}$ and $\mathbf{r}_{2}$ were separated by one meter, since if $\mathbf{r}_{1}$ is in a planet, $\mathbf{r}_{2}$ probably is as well. $\psi$ would nonethe- 
less be translationally invariant, corresponding to superpositions of planets at different locations, with all locations given equal weight. In summary, non-linear dynamics with chaotic behavior will create a type of hidden complexity within the wavefunction $\psi$, but not itself cause symmetry breaking. In the following section we will argue that the symmetry breaking is apparent, as well as discuss why it appears to be broken in a way that favors "classical" states.

\section{UNIVERSALLY VALID QUANTUM MECHANICS}

\subsection{A Brief Review of the No Collapse Interpretation}

Although quantum mechanics has proven to be an astonishingly successful theory by any standards, the issue of how it is to be interpreted has caused heated debate ever since it was created some 70 years ago. At the center of the controversy stands the so called collapse postulate, according to which the time-evolution of the wavefunction is occasionally not governed by the Schrödinger equation, but described by a discontinuous random jump, a socalled "collapse". This postulate was introduced to explain the apparently random outcomes of certain quantum measurements, but is completely unnecessary according to the Everett interpretation [23 28], also known as the "many worlds interpretation". The debate continues about the underlying philosophical and ontological issues (see, e.g., 29, 31]), but these are not crucial to the argument in this paper. All that is needed here is the assumption that quantum mechanics is universally valid:

- The time-evolution of any isolated system is governed by the Schrödinger equation.

This is what Albert refers to as the "raw theory". Thus all isolated systems, not merely microscopic ones, are assumed to obey quantum mechanics, be they planets, human beings or the entire universe. In other words, there is no wave function collapse built into the theory itself. Rather, this phenomenon is assumed to arise automatically on a phenomenological level. Everett argued that a generic observer will subjectively experience an $a p$ parent wavefunction collapse consistent with the familiar quantum probabilities. The basic idea is illustrated by the following simple example. Suppose Angélica prepares $n$ different spin- $\frac{1}{2}$ silver atoms to have spin up in the $x$ direction, and then proceeds to measure their spin in the $z$-direction with a Stern-Gerlach apparatus. She writes down the results in the form of binary digits, 0 denoting "down" and 1 denoting "up", with a decimal point in front of it all, so that the outcome of the entire set of measurements can be codified as a real number $x$ on the interval $[0,1]$. For instance, if $n=4$, the outcome "up-down-down-up" would correspond to $x=0.1001$. In the limit where $n \rightarrow \infty$, there is thus a one-to-one correspondence between outcomes and real numbers on $[0,1]$. In the Everett interpretation, the final state for 
the system consisting of both Angélica, her laboratory and her silver atoms is thus a superposition of orthogonal states that we can label by numbers $x$, such that state $x$ corresponds to the atoms having precisely the spins given by the decimals of $x$ and Angélica having experienced exactly these measurement results. The key point realized by Everett is that almost all Angélicas in this final superposition will have measured spin up 50\% of the time, in exactly the same sense that almost all real numbers on $[0,1]$ have $50 \%$ of their binary digits equal to one (the set of those that do not has Lebesgue measure zero). Thus almost all Angélicas in the final superposition will find the outcome of their experiment in agreement with the standard quantum probabilities. The generalization to finite $n$ and more generic probabilities than $50 \%$ is also presented by Everett, and in every case, he finds that almost all observers in the final superposition will find their experimental results in agreement with the probabilities that would arise from the collapse hypothesis. Certain aspects of Everett's arguments are still controversial, but as mentioned, these technical details are of no importance for the main argument in the present paper.

\subsection{How Universal Quantum Mechanics Causes Apparent Complexity}

The above example brings us back to the argument of Section 2.1, except that we are now discussing reality 2 rather than physical models. To rephrase the argument in quantum language, there is relatively little algorithmic information in the final state $\psi$. To describe any one particular outcome in the grand superposition, however, would require $n$ bits of information, and we can for arguments sake choose $n$ to be enormous. Thus although reality $(\psi)$ remained as simple as it was before the experiment, a generic Angélica in the final superposition will be staring at a piece of paper with a long and seemingly random string zeroes and ones and consider this to be lots of information. Thus subjectively, as experienced by a self-aware subset of $\psi$ (Angélica), the complexity and algorithmic information of the world has increased, although objectively, when viewing the mathematical object $\psi$ from outside, the algorithmic information of $\psi$ stayed constant.

This is precisely what happens in the example of cosmological structure formation discussed in the previous section. Even if nonlinear dynamics produces a wavefunction $\psi$ where a planet is in a superposition of being centered in two different places, separated by one meter, this is of course not

\footnotetext{
${ }^{2}$ In this paper, we use the word "reality" to denote that which is described by $\psi$. In other words, we think of all elements of the a superposition as being equally real, bearing in mind that in any particular element of the superposition, there may be an observer who subjectively feels that merely that element is real. (This is of course merely an issue of semantics, so all that matters is that we clearly state how we choose to use various words, deferring to philosophers the question of which usage is preferable.)
} 
what a self-aware subset of $\psi$ would experience. Rather, after the observer had interacted with the planet (by absorbing electromagnetic radiation reflected from it, say), $\psi$ would for all practical purposes be a superposition of two orthogonal states, each corresponding to the planet having a definite location and the observer finding it in that same location. In other words, when we gaze into the night sky and see stars all over the place (in definite positions, but positions that appear somewhat random), this is exactly what we would have expected to see. However, in the picture put forward here, the seemingly large information content in these stellar positions (information that has by now filled many volumes of astronomy publications) is merely information in the eyes of the beholder. It is not real information in the algorithmic sense, and would never enter in a compact description of $\psi$ itself.

\subsection{The Role of Decoherence}

The no collapse interpretation of quantum mechanics leaves a crucial question unanswered [30,31,32]: why is the position operator $\widehat{\mathbf{r}}$ so special? In other words, why is it that we tend to find macroscopic objects in (approximate) eigenstates of $\widehat{\mathbf{r}}$ in particular, even though there are infinitely many other Hermitean operators (for instance $\widehat{\mathbf{r}}+\widehat{\mathbf{p}}$ ) whose eigenstates involve macro-superpositions (such as the above-mentioned superposition of a planet being in two macroscopically distinct places)? A breakthrough on this issue was not made until more than a decade later [33], and it took another decade until the question was definitely answered [34,35]. The answer, usually referred to as decoherence (see [36] for a recent review), lies in the fact that macroscopic systems are virtually impossible to isolate from their surroundings. It can be shown that the net effect of this inevitable interaction is to suppress off-diagonal elements of the spatial density matrix, which means that to a subjective observer, a macroscopic object will for all practical purposes appear to have a definite position. [3 It is straightforward to quantify the decoherence effect caused by various interactions that we know to occur [37,38, and one finds that even such relatively weak effects as scattering of microwave background photons and (on earth) solar neutrinos will keep an object the size of a bowling ball localized to within a small fraction of an atomic radius.

Decoherence is quite crucial to the main argument in this paper. If Angélica had measured the spin of her silver atoms in the $x$-direction instead, she would simply have measured "up" every time. The quantum creation of apparent complexity that was described above of course requires that we are measuring an observable that the quantum system is not already in an eigenstate of. Thanks to decoherence, our classical world is very posi-

\footnotetext{
${ }^{3}$ The deeper question of why the calculations always end up favoring the position operator is related to the unexplained empirical fact that the Lagrangians of nature seem to be spatially local (as opposed to being local in for instance Fourier space).
} 
tion centered, so our minds are constantly keeping track of where things are located. As we saw in the previous section, nonlinear gravitational evolution will automatically convert a translationally invariant initial state into a state where macroscopic objects are not in position eigenstates, but rather in a superposition of being in many different places. This is why we should expect to find apparent complexity in the sky. Electromagnetic, weak and strong forces of course all produce non-linear time-evolution as well, so we have no right to be surprised by finding apparently complex structures on more everyday scales either - after all, non-linear dynamics is so ubiquitous that it has been jocularly compared to "the zoology of non-elephants".

\section{DISCUSSION}

We have argued that although the universe appears to contain an enormous, perhaps even infinite, amount of information, this impression is nonetheless consistent with the assumption that its algorithmic information content is quite small, even if no new physics whatsoever is invoked. In short, reality could in fact be much simpler than it appears, with its apparent plethora or complex structures such as trees, fountains and rabbits. The enormous discrepancy between apparent and actual information content would have been caused by an interplay of several well-known effects:

- Non-linear dynamics evolved quantum states that were initially only microscopically different into quantum states that were macroscopically different.

- Well-known decoherence effects ensured that only macroscopically "classical" states could be perceived by self-aware subsets of the universe (such as us), so for all practical purposes, the wavefunction of the universe is in a superposition of a such states.

- Although the total wavefunction still contains as little algorithmic information as it did in the beginning (assuming that the laws of physics that govern its time evolution are known), any one element of this superposition (which is all that we can subjectively experience, because of decoherence) will display the enormous complexity brought about by the chaotic non-linear evolution.

Thus even if both the laws of physics and the initial conditions exhibit so much symmetry that they are easy to describe, we would not expect most of these symmetries to be manifested in what we see when we open our eyes. For instance, we would not expect the matter distribution around us to be translationally invariant even if the initial wave function was. 


\subsection{Why Universal Quantum Mechanics is Needed}

It should be emphasized that if the wavefunction really collapses, then the main argument of this paper does as well. In other words, in those interpretations of quantum mechanics where the Schrödinger evolution is replaced by some random process while Angélica measures her spins, leaving a final wavefunction $\psi$ corresponding to merely one definite sequence of outcomes, the algorithmic information of $\psi$ will in fact increase. The reason that it is no longer conserved is of course the randomness itself, since causality has been lost and the initial data no longer suffice to specify the final wavefunction. Rather, the randomness will increase the algorithmic information with one bit per spin measurement.

\subsection{Why Chaos is Needed}

Why does the above argument fail in the absence of non-linearf dynamics? Roughly speaking, the apparent symmetry breaking caused by quantum mechanics and decoherence applied only to macro-superpositions, whereas the only superpositions that are required to exist initially by the Heisenberg uncertainty principle is micro-superpositions. Thus for our argument to work, we need some process to evolve the initial micro-superpositions into macro-superpositions. In our example of cosmic structure formation, we needed a mechanism that converted the microscopic density fluctuations of the early universe into planets etc. that were in superpositions of being in many macroscopically separated positions at once. Converting micro into macro is of course the very essence of chaotic dynamics, which exponentially amplifies microscopic differences in initial data as time passes.

\subsection{What about Computability?}

In the definition of algorithmic information, the universal computer is of course required to produce the desired output in a finite amount of time. In other words, the current wavefunction of the universe should be computable from the initial state and the equation of motion, to any prescribed accuracy, in a finite number of program steps, As long as the equations of motion are non-chaotic, this will be achievable by integrating the partialdifferential equations of motion numerically, using some time-step $\Delta t$ that is

\footnotetext{
${ }^{4}$ Here and throughout we refer to a system as linear if its Hamiltonian is at most quadratic. This corresponds to the classical equations of motion in phase space being linear. Thus the phase-space distributions at two different times are simply related by a linear canonical transformation. For such quantum systems, the Wigner functions at two different times are related by this same linear canonical transformation. This type of linearity should not be confused with that of the Schrödinger equation, which is of course linear (in $\psi$ ) however nonlinear the Hamiltonian may be.
} 
chosen small enough to fulfill the accuracy requirement. If the equations of motion are chaotic, however, this simple-minded approach of course fails in practice, since the computed final state would be significantly wrong unless an absurdly high numerical precision was used, the number of significant digits required growing exponentially with the duration between the initial and final time. Fortunately, the relevant quantity in our case is the universal wave-function $\psi$, whose evolution is non-chaotic, since it is governed by the linear Schrödinger equation. Thus ironically, although the chaotic nature of the corresponding classical dynamics was crucial to a different aspect of our argument, the non-chaotic nature of pure quantum evolution is important as well.

In summary, whereas quantum states themselves evolve non-chaotically, the reduced density matrices describing subsystems do evolve chaotically, and both of these formal facts are essential to our argument.

\subsection{What about the Initial State?}

It should be emphasized that although we assumed that the initial state of the universe had quite a small algorithmic information content, our argument about apparent complexity holds more generally, in the sense that an observer will always perceive the universe to be more complicated than it actually is. Roughly speaking, the observer's description (as given by the appropriate reduced density matrix) will inherit all the complexity of the big bang, and in addition include substantial amounts of extra "illusory" complexity.

\subsection{What about Entropy?}

The subtle relationship between entropy and various measures of information has been discussed by many authors (see e.g. [16, 17, 40] for reviews), often in connections with Maxwell's infamous demon. It has been emphasized [39] that neither a state of minimum disorder (such as a perfect crystal) nor maximum disorder (such as an ideal gas in thermal equilibrium) corresponds to what we with a course-grained description would describe as complex. Rather, the hallmark of perceived complexity is the so called "depth", which often reduces to the difference between the coarse-grained and fine-grained entropies [39]. This provides an alternative way summarizing the arguments above: the fine-grained entropy of the universe remains constant, but non-linear dynamics and the apparent symmetry-breaking brought about by quantum mechanics and decoherence increase the coarsegrained entropy that we perceive, thus increasing the depth, the apparent complexity.

In addition to these processes, the course-grained entropy of any open system will of course increase in accordance with the second law of thermodynamics, even in classical physics, as the system gradually becomes correlated with ever more distant parts of the universe - see e.g. [40] for 
a recent review, including a discussion of when the entropy can decrease. However, such an entropy increase merely reflects an increase in our ignorance about the system in question, not an increase in the complexity of the system. Consider a visible particle suspended in a static liquid, undergoing Brownian motion. If we observe it at some definite position, and then look away from our microscope, our subjective probability distribution for the particle position will widen over time as $\sqrt{t}$. Yet when we eventually look again, thereby reducing our ignorance and thus reducing the entropy, the system we see appears neither more nor less complex than it did to begin with.

\subsection{Would it be Testable?}

Is this entire discussion merely a useless metaphysical digression without any experimentably testable consequences? Not necessarily. Ever since the big bang theory became firmly established, the problem of understanding the initial conditions has aroused interest. For instance, one of the main goals of modern cosmology is to accurately measure the power spectrum of density fluctuations. By comparing the fluctuations measured by large galaxy redshift surveys to the fluctuations measured in the cosmic microwave background radiation, strong constraints can be placed on models for the formation of large scale structure in the universe. Most of the currently popular models (cold dark matter, mixed dark matter, baryonic dark matter, etc.) simply predict how a "primordial" power spectrum would evolve due to various microphysical effects, which means that all these models must be supplemented with an assumption about the form of this primordial spectrum. One popular prediction of what the primordial power spectrum should be is the inflationary scenario (see e.g. [19 for a review), but many other suggestions have been put forward as well, including ones appealing to quantum gravity, e.g. 41,42. The point is that the initial conditions prevailing shortly after the big bang are not necessarily in the realm of metaphysics, and may turn out to have measurable consequences. If this turns out to be the case, then it will perhaps be possible for us to use our knowledge of the mechanisms described above to make inferences about the initial conditions from observational data, and if these initial conditions are indeed simple enough to have a small information content, we may even be able to determine them with reasonable accuracy.

\subsection{Would it be Good News or Bad News?}

A pessimistic way of summarizing the scenario put forward in this paper would be as yet another step in the human humiliation that began with the Copernican revolution. After being dethroned from our position in the center of the universe half a millennium ago, forced to accept that we are orbiting the sun rather than vice versa, our human egos have received a series of devastating blows. We have now been demoted further, to orbiting 
a rather average star a rather average distance from the center of a rather average galaxy, our sun being but one star amongst $10^{11}$ others in but one galaxy amongst at least that many more. Alas, the humiliation shows no signs of abating. In the cold dark matter scenario and variants thereof, the bulk of the matter in the universe is conjectured to be non-baryonic, snobbishly refusing to interact with us much at all, leaving us as some sort of ignored fringe minority. Our human self-confidence has been deflated on the mental side as well. Darwin told us that we were descended from amoebas and differed from other organisms not in a qualitative way but merely in a quantitative way. Freud claimed that we did not even understand ourselves all that well, and Skinner argued that we had no free will, being more like organic computers that could be programmed by suitable conditioning. Last

year, Garri Kasparov was beaten by a chess computer. Now we are arguing that even the intricate structures that are the subjects of our thoughts, dreams and efforts in life, everything from our loved ones to our parking tickets, are perhaps more aptly described as illusions, as manifestations of the fact that our minds experience the grandeur of reality merely from an extremely limited frog perspective. More precisely, we are arguing that it is the grandeur itself that is merely apparent, since the complete description of the wavefunction of the universe is orders of magnitude simpler than what we see when we open our eyes. In short, reality would be much more banal than it appears to be.

However, we can just as well choose to summarize our discussion on an optimistic note. First of all, once we have abandoned the notion of us humans playing a central role in the universe, and forced ourselves to find a raison d'être elsewhere, yet another step away from the limelight clearly makes little difference. The everyday reality to which we wake up in the mornings will of course remain the same whether we change our ontological views or not - no amount of philosophizing will make those parking tickets go away. If we thus restrict our value judgements to empirical considerations, the picture put forward here would have quite positive implications for our ability to do science in the future. The information content of the world around us naively appears to completely dwarf the sort of information quantities that we humans can store and process. However, if the algorithmic information content of the universe is indeed quite small, then perhaps we need not despair when faced with the vast and complex world around us. Rather, this would raise the slight hope that we humans may one day be able to understand it.

\section{REFERENCES}

[1] H. Yukawa, Prog. Theo. Phys. Suppl. 37\&38, 512 (1966).

[2] H. Yamamoto, Phys. Rev. D 30, 1727 (1984).

[3] K. E. Plokhotnikov, Sov. Phys. Doklady 36, 38 (1991).

[4] R. J. Solomonoff, Inf. Control 7, 1 (1964). 
[5] A. N. Kolmogorov, Inf. Transmission 1, 3 (1965).

[6] A. N. Kolmogorov, IEEE Trans. Inf. Theory 14, 662 (1968).

[7] A. N. Kolmogorov, Usp. Mat. Nauk 25, 602 (1970).

[8] G. J. Chaitin, Ass. Comput. Mach. 13, 547 (1966).

[9] G. J. Chaitin, Scient. Am. 232(5), 47 (1975).

[10] G. J. Chaitin, Ass. Comput. Mach. 22, 245 (1975).

[11] G. J. Chaitin, IBM J. Res. Dev. 21, 350 (1977).

[12] G. J. Chaitin, Algorithmic Information Theory, (Cambridge University Press, Cambridge, 1987).

[13] P. Gacs, P. Soviet Math. Dokl. 15, 1477 (1974).

[14] L. A. Levin, Soviet Math. Dokl. 17, 522 (1976).

[15] I. Stewart, Nature 332, 115 (1988).

[16] W. H. Zurek, Nature 341, 119 (1989).

[17] W. H. Zurek, Phys. Rev. A 40, 4731 (1989).

[18] C. M. Caves, Phys. Rev. E 47, 4010 (1993).

[19] E. Kolb and M. S. Turner, The Early Universe (Addison-Wesley, Reading, 1990).

[20] E. P. Wigner, Phys. Rev. 40, 749 (1932).

[21] Y. S. Kim \& M. E. Noz, Phase Space Picture of Quantum Mechanics: Group Theoretical Approach (World Scientific, Singapore, 1991).

[22] W. H. Zurek \& J. P. Paz, Phys. Rev. Lett. 72, 2508 (1994).

[23] H. Everett III, Rev. Mod. Phys. 29, 454 (1957).

[24] H. Everett III, The Many-Worlds Interpretation of Quantum Mechanics, B. S. DeWitt and N. Graham, eds. (Princeton University Press, Princeton, 1986).

[25] J. A. Wheeler, Rev. Mod. Phys. 29, 463 (1957).

[26] L. M. Cooper \& D. van Vechten, Am. J. Phys. 37, 1212 (1969).

[27] L. N. Cooper, "Wave function and observer in quantum theory", in The Physicist's Conception of Nature, J. Mehra, ed. (Reidel, Dordrecht, 1983).

[28] B. S. DeWitt, Phys. Today 23 (9), 30 (1971).

[29] A. Peres \& W. H. Zurek, Am. J. Phys. 50, 807 (1982).

[30] Y. Ben-Dov, Found. Phys. 3, 383 (1990).

[31] A. Kent, Int. J. Mod. Phys. 5, 1745 (1990).

[32] E. J. Squires, Found. Phys. Lett. 3, 87 (1990).

[33] H. D. Zeh, Found. Phys. 1, 69 (1970). 
[34] W. H. Zurek, Phys. Rev. D 24, 1516 (1981).

[35] W. H. Zurek, Phys. Rev. D 26, 1862 (1982).

[36] W. H. Zurek, Phys. Today 44 (10), 36 (1991).

[37] E. Joos and H. D. Zeh, Z. Phys. B 59, 223 (1985).

[38] M. Tegmark, Found. Phys. Lett. 6, 571 (1993).

[39] S. Lloyd \& H. Pagels, Annals of Phys. 188, 186 (1988).

[40] S. Lloyd, Phys. Rev. A 39, 5328 (1989).

[41] S. W. Hawking, Nucl. Phys. B 239, 257 (1984).

[42] T. Souradeep, ApJ 402, 375 (1993). 